\title{
Obstrução Coronária Após Implante de Válvula Aórtica por Cateter para o Tratamento de Bioprótese Valvular Cirúrgica com Disfunção: Revisão Sistemática da Literatura
}

Henrique Barbosa Ribeiro', Luis Nombela-Franco ${ }^{2}$, Ricardo Allende ${ }^{3}$, Marina Urena ${ }^{4}$, Ignacio Amat-Santos ${ }^{5}$, Sergio Pasian ${ }^{6}$, Daniel Doyle ${ }^{7}$, Robert DeLarochellière ${ }^{8}$, Mélanie Côté ${ }^{9}$, Éric Dumont ${ }^{10}$, Josep Rodés-Cabau ${ }^{11}$

\section{RESUMO}

Introdução: Há poucos dados na literatura que avaliam a obstrução coronária após implante transcateter de válvula aórtica (TAVI, do inglês transcatheter aortic valve implantation) para o tratamento de disfunção de bioprótese aórtica (valve-in-valve ViV). O presente estudo avaliou, por meio de revisão sistemática da literatura, as características clínicas, o manejo e os desfechos clínicos de pacientes com obstrução coronária após TAVI-ViV. Métodos: Estudos publicados entre 2002 e 2013 avaliando a obstrução coronária como complicação de TAVI-ViV foram identificados por meio de busca eletrônica sistemática. Foram avaliados dados basais sobre as características clínicas e do procedimento, manejo da complicação e desfechos clínicos. Resultados: Foram identificadas, no total, quatro publicações descrevendo sete pacientes. A maioria dos pacientes era do sexo feminino $(71 \%)$, sendo a média de idade de $82 \pm 5$ anos, com STS-PROM de 9,4 $\pm 2,6 \%$. As médias da altura do tronco da artéria coronária esquerda (TCE) e o diâmetro médio da raiz aórtica foram de 8,8 $\pm 1,5 \mathrm{~mm}$ e 28,0 \pm 5,0 mm, respectivamente. A maioria dos pacientes apresentava biopróteses com suporte e folhetos montados externamente, ou eram próteses sem suporte (stentless) e o TCE foi envolvido em todos os casos. A intervenção coronária percutânea (ICP) foi tentada em todos os pacientes, tendo sucesso em quatro deles (57\%). A mortalidade intra-hospitalar foi $42,9 \%$ (três casos), todos após ICP sem sucesso. Conclusões: A obstrução coronária após TAVI-ViV ocorreu mais frequentemente em mulheres com bioprótese com suporte e folheto montado externamente ou com bioprótese sem suporte. O TCE foi envolvido em todos

\section{ABSTRACT}

\section{Coronary Obstruction Following Transcatheter Aortic Valve Implantation for Degenerative Bioprosthetic Surgical Valves: a Systematic Literature Review}

Background: Very few data exist on coronary obstruction following transcatheter aortic valve implantation (TAVI) for degenerative bioprosthetic valves (valve-in-valve - ViV). The present study evaluated, through a systematic review of the literature, the clinical characteristics, management and clinical outcomes of patients with coronary obstruction after ViV-TAVI. Methods: Studies published between 2002 and 2013 evaluating coronary obstruction as a complication of ViV-TAVI were identified using a systematic electronic search. Data on the clinical and procedural characteristics, management of the complication, and clinical outcomes were analyzed. Results: A total of four publications describing seven patients were identified. Most patients $(71 \%)$ were women, with mean age of $82 \pm 5$ years, and STS-PROM score of $9.4 \pm 2.6 \%$. Mean left coronary artery (LCA) ostium height and aortic root width were $8.8 \pm 1.5 \mathrm{~mm}$ and $28.0 \pm 5.0 \mathrm{~mm}$, respectively. Most patients had stented bioprosthetic valves with externally mounted leaflets or stentless aortic bioprosthesis, and the LCA was involved in all patients. Percutaneous coronary intervention (PCI) was attempted in all patients and was successful in four (57\%). In-hospital mortality was $42.9 \%$ (three cases), all of them after failed $\mathrm{PCl}$. Conclusions: Coronary obstruction following ViV-TAVI occurred more frequently in women with stented

\footnotetext{
Pós-Graduando (Doutorado) pela Quebec Heart \& Lung Institute, Laval University. Quebec, Canadá.

${ }^{2}$ Fellow do Quebec Heart \& Lung Institute, Laval University. Quebec, Canadá.

${ }^{3}$ Fellow do Quebec Heart \& Lung Institute, Laval University. Quebec, Canadá.

4 Pós-Graduanda (Doutorado) pelo Quebec Heart \& Lung Institute, Laval University. Quebec, Canadá.

${ }^{5}$ Fellow do Quebec Heart \& Lung Institute, Laval University. Quebec, Canadá.

${ }^{6}$ Radiologista Chefe do Quebec Heart \& Lung Institute, Laval University. Quebec, Canadá.

${ }^{7}$ Cirurgião Cardíaco do Quebec Heart \& Lung Institute, Laval University. Quebec, Canadá.
}

\footnotetext{
${ }^{8}$ Chefe da Cardiologia do Quebec Heart \& Lung Institute, Laval University. Quebec, Canadá.

${ }^{9}$ Coordenadora do Centro de Pesquisas em Cardiopatias Estruturais do Quebec Heart \& Lung Institute, Laval University. Quebec, Canadá. ${ }^{10}$ Cirurgião Cardíaco Chefe do Programa de TAVI do Quebec Heart \& Lung Institute, Laval University. Quebec, Canadá.

11 Chefe do Serviço de Hemodinâmica do Quebec Heart \& Lung Institute, Laval University. Quebec, Canadá.
}

Correspondência: Henrique Barbosa Ribeiro. Avenida São Gabriel, 201 sala 1.304 - São Paulo, SP, Brasil - CEP 01435-001 E-mail: henriquebribeiro@me.com

Recebido em: 14/9/13 - Aceito em: 24/11/13 
os casos e a ICP foi realizada com sucesso em $60 \%$ deles. Esforços contínuos poderão auxiliar na detecção dos fatores associados a essa complicação, no intuito de se implementarem medidas apropriadas para sua prevenção.

DESCRITORES: Valva aórtica. Implante de prótese de valva cardíaca. Oclusão coronária.

0 implante transcateter de válvula aórtica (TAVI, do inglês transcatheter aortic valve implantation) tem sido estabelecido como padrão para o tratamento de pacientes com estenose aórtica grave sintomática considerados inoperáveis e como uma alternativa para aqueles considerados de alto risco para troca valvar aórtica cirúrgica. ${ }^{1,2}$ No entanto, o TAVI também tem sido associado a complicações bastante raras, porém muitas vezes fatais, como a obstrução coronária, que é geralmente relacionada ao deslocamento dos folhetos nativos calcificados da válvula em direção aos óstios coronários.

Nós realizamos recentemente uma revisão sistemática da literatura sobre a obstrução coronária sintomática, como uma complicação do TAVI, que incluiu um total de 24 casos, todos eles relatados isoladamente como relatos de caso ou séries pequenas de casos.. ${ }^{3}$ Tal revisão demonstrou que a obstrução coronária, após $\mathrm{TAVI}$, ocorreu mais frequentemente em mulheres e em pacientes que receberam uma válvula expansível por balão, sendo o tronco da artéria coronária esquerda (TCE) a artéria mais frequentemente envolvida. Entretanto, este estudo excluiu procedimentos em pacientes com bioprótese cirúrgica prévia (valve-in-valve - ViV). Além disso, recente registro multicêntrico, que incluiu mais de 6.500 pacientes, sugeriu que essa complicação poderia ser até quatro vezes mais frequente em pacientes portadores de biopróteses cirúrgicas, de forma semeIhante aos dados relatados no registro global de ViV. ${ }^{4}$

No entanto, nenhum estudo até hoje avaliou especificamente essa complicação na população ViV. Os objetivos do presente estudo foram, dessa forma, avaliar as características basais, o manejo e os resultados clínicos da obstrução coronária como complicação do TAVI em pacientes com bioprótese aórtica cirúrgica prévia, por meio de uma revisão sistemática de todos os estudos publicados até o momento.

\section{MÉTODOS}

Todos os artigos relevantes em inglês sobre TAVI e obstrução coronária em pacientes com bioprótese cirúrgica anterior, publicados entre dezembro de 2002 e setembro de 2013, foram pesquisados sistematicamente no BioMedCentral (http://www.biomedcentral.com), no Google Scholar (http://www.scholar.google.com) e no PubMed (http://www.pubmed.gov). Os seguintes termos de consulta foram usados: "aortic stenosis", "transcatheter aortic valve implantation", "transcatheter bioprosthetic valves with externally mounted leaflets or with stentless bioprosthesis. The LCA was involved in all cases and $\mathrm{PCl}$ was successful in $60 \%$ of them. Continued efforts may help identify the factors associated with this complication so that appropriate prevention measures may be implemented.

DESCRIPTORS: Aortic valve. Heart valve prosthesis implantation. Coronary occlusion.

aortic valve replacement", "transcatheter heart valve", "heart valve prosthesis implantation", "coronary stenosis", "coronary occlusion, coronary obstruction", "prior surgical bioprosthesis", e "valve-in-valve". Outros estudos foram avaliados por meio de pesquisa manual de fontes secundárias, incluindo referências de artigos primários (backward snowballing) e contatos com especialistas internacionais.

As citações foram selecionadas com base no título/ resumo por dois revisores independentes (HBR e LNF) e recuperadas como manuscritos completos se consideradas potencialmente pertinentes. As divergências foram resolvidas após consenso, para reunir todos os relatos e séries de casos pertinentes sobre obstrução coronária após TAVI-ViV. Os artigos publicados, que incluíram apenas a incidência da complicação, foram excluídos desta análise.

Os dados reunidos incluíram as características clínicas basais, além das ecocardiográficas e de tomografia computadorizada (TC). As variáveis da TC incluíram dados de altura do TCE em relação ao ânulo aórtico e dos diâmetros da raiz e ânulo aórtico. Dados do procedimento, como tipo e tamanho da válvula transcateter utilizada, tipo de abordagem, quadro clínico e manejo da obstrução coronária também foram avaliados. Finalmente, dados sobre a mortalidade intra-hospitalar também foram recuperados.

Variáveis categóricas foram relatadas como n (\%) e variáveis contínuas como média \pm desvio padrão. Todas as análises foram realizadas usando o pacote estatístico SAS, versão 9.3 (SAS Institute Inc, Cary, NC, Estados Unidos).

\section{RESULTADOS}

Um total de quatro publicações foi identificado, descrevendo, por sua vez, um total de sete pacientes que tiveram obstrução coronária relacionada ao procedimento TAVI-ViV. ${ }^{5-8}$ Todos os estudos se referiram a relatos de casos, exceto um estudo multicêntrico, que incluiu três casos de obstrução coronária. As principais características clínicas basais estavam disponíveis para todos os pacientes. Dados de TC sobre a altura do óstio do TCE e do ânulo aórtico e medidas da raiz aórtica foram relatados em quatro, quatro e três pacientes, respectivamente. Dados clínicos e do procedimento sobre a apresentação clínica, diagnóstico e manejo da obstrução coronária estiveram disponíveis em todos os 
pacientes. Todos os estudos relataram dados sobre os desfechos intra-hospitalares.

As principais características clínicas, ecocardiográficas, da TC e dos procedimentos aos quais os pacientes foram submetidos são evidenciadas nas Tabelas 1 (dados individuais) e 2 (médias). A média de idade da população estudada foi de $82 \pm 5$ anos, sendo, em sua maioria, constituída por mulheres $(71,4 \%)$. Dados da TC revelaram média da altura do TCE de $8,8 \pm 1,5 \mathrm{~mm}$ e o diâmetro médio da raiz aórtica de 28,0 $\pm 5,0 \mathrm{~mm}$. A válvula expansível por balão Edwards ${ }^{\circledR}$ (Edwards Lifesciences, Irvine, CA) foi usada em cinco pacientes e uma válvula autoexpansível CoreValve ${ }^{\circledR}$ (Medtronic, Minneapolis, MN) foi usada em dois pacientes.

Os dados principais sobre quadro clínico e conduta adotada em relação à obstrução coronária são mostrados nas Tabelas 3 (dados individuais) e 4 (médias). O TCE foi envolvido em todos os pacientes (em um deles, junto da artéria coronária direita); todos os pacientes apresentaram hipotensão importante e mantida, e a maioria deles $(57,1 \%)$ apresentou alterações eletrocardiográficas do segmento ST. O diagnóstico de obstrução coronária foi feito por angiografia coronária em todos os pacientes e a obstrução estava relacionada ao deslocamento dos folhetos da bioprótese em todos eles.

A intervenção coronária percutânea (ICP) foi tentada em todos os pacientes e teve sucesso em quatro deles $(57,1 \%)$. Os três casos de insucesso da ICP ocorreram por: impossibilidade de atravessar a obstrução com o fio-guia coronário, necessitando de cirurgia de revas- cularização miocárdica de urgência; impossibilidade de restabelecer o fluxo coronário, a despeito do im-

TABELA 2

Características basais clínicas, ecocardiográficas, da tomografia computadorizada e do procedimento da população estudada

\begin{tabular}{lc}
\hline Variáveis clínicas & (n = 7) \\
\hline Idade, anos & $82,3 \pm 4,6$ \\
Sexo feminino, $\mathrm{n}(\%)$ & $5(71,4)$ \\
Classe NYHA III-IV, $\mathrm{n}(\%)$ & $6(85,7)$ \\
Cirurgia de revascularização miocárdica prévia, $\mathrm{n}(\%)$ & $4(57,1)$ \\
Escore STS-PROM, \% & $9,4 \pm 2,6$ \\
Dados ecocardiográficos e de tomografia computadorizada & \\
Gradiente aórtico médio, mmHg & $42,2 \pm 28,1$ \\
Área da válvula aórtica, cm ${ }^{2}$ & $0,43 \pm 0,09$ \\
Ânulo aórtico, mm & $19,1 \pm 1,8$ \\
Altura do tronco da artéria coronária esquerda, mm & $8,8 \pm 1,5$ \\
Diâmetro da raiz aórtica, mm & $28,0 \pm 5,0$ \\
Dados do procedimento & \\
Abordagem, $\mathrm{n}$ (\%) & \\
$\quad$ Transfemoral & $6(85,7)$ \\
$\quad$ Transapical & $1(14,3)$ \\
Tipo de válvula, $\mathrm{n}$ (\%) & \\
$\quad$ Sapien ${ }^{\circledR}$ e Sapien XT & \\
CoreValve & \\
\hline NYHA = New York Heart Association. & $5(71,4)$ \\
\hline
\end{tabular}

TABELA 1

Características clínicas basais e do procedimento

\begin{tabular}{|c|c|c|c|c|c|c|c|c|c|c|c|c|c|c|}
\hline Estudo & $\begin{array}{l}\text { Idade } \\
\text { (anos) }\end{array}$ & Sexo & $\begin{array}{c}\text { Tipo de } \\
\text { bioprótese }\end{array}$ & $\begin{array}{c}\text { Nome da } \\
\text { bioprótese } \\
\text { cirúrgica }\end{array}$ & $\begin{array}{c}\text { Tamanho } \\
\text { da } \\
\text { bioprótese } \\
(\mathrm{mm})\end{array}$ & $\begin{array}{l}\text { CRM } \\
\text { prévia }\end{array}$ & $\begin{array}{l}\text { Escore } \\
\text { STS (\%) }\end{array}$ & $\begin{array}{l}\text { Gradiente } \\
\text { aórtico } \\
\text { médio } \\
(\mathrm{mmHg})\end{array}$ & $\begin{array}{c}\text { Ânulo } \\
\text { aórtico } \\
\text { (mm) }\end{array}$ & $\begin{array}{c}\text { Raiz } \\
\text { aórtica } \\
\text { (mm) }\end{array}$ & $\begin{array}{c}\text { Altura } \\
\text { TCE } \\
(\mathrm{mm})\end{array}$ & Acesso & Tipo de TAVI & $\begin{array}{c}\text { Tamanho } \\
\text { TAVI (mm) }\end{array}$ \\
\hline $\begin{array}{l}\text { Fiorina } \\
\text { et al. }{ }^{5}\end{array}$ & 82 & M & $\begin{array}{l}\text { Sem suporte } \\
\text { (stentless) }\end{array}$ & $\begin{array}{l}\text { CryoLife- } \\
\text { 0'Brien }{ }^{\circledR}\end{array}$ & 25 & Não & - & - & - & 33 & 10 & TF & CoreValve $^{\circledR}$ & 29 \\
\hline $\begin{array}{l}\text { Gurvitch } \\
\text { et al. }^{6}\end{array}$ & 86 & $\mathrm{~F}$ & Com suporte & Mitroflow $^{\circledR}$ & 21 & Sim & 12,9 & 87 & 18 & - & - & $\mathrm{TA}$ & Sapien $^{\circledR}$ & 23 \\
\hline $\begin{array}{l}\text { Gurvitch } \\
\text { et al. }{ }^{6}\end{array}$ & 78 & $\mathrm{~F}$ & Com suporte & Mitroflow ${ }^{\circledR}$ & 21 & Sim & - & 44 & - & - & - & TF & CoreValve $^{\circledR}$ & 26 \\
\hline $\begin{array}{l}\text { Chakravarty } \\
\text { et al. }{ }^{7}\end{array}$ & 79 & $\mathrm{~F}$ & $\begin{array}{l}\text { Sem suporte } \\
\text { (stentless) }\end{array}$ & $\begin{array}{l}\text { St. Jude } \\
\text { Toronto } \\
\text { SPV }^{\circledR}\end{array}$ & 23 & Não & 6,1 & - & - & - & 7,4 & TF & Sapien $^{\circledR}$ & 23 \\
\hline $\begin{array}{l}\text { Ribeiro } \\
\text { et al. }{ }^{8}\end{array}$ & 80 & $\mathrm{~F}$ & $\begin{array}{l}\text { Sem suporte } \\
\text { (stentless) }\end{array}$ & $\begin{array}{c}\text { St. Jude } \\
\text { Toronto } \\
\text { SPV }^{\circledR}\end{array}$ & 24 & Não & 8,1 & 22 & 20 & 23,2 & 9,7 & TF & Sapien $^{\circledR}$ & 23 \\
\hline $\begin{array}{l}\text { Ribeiro } \\
\text { et al. }{ }^{8}\end{array}$ & 91 & $\mathrm{~F}$ & Com suporte & $\begin{array}{l}\text { Carpentier- } \\
\text {-Edwards }\end{array}$ & 21 & Sim & 10,3 & 43 & 18 & - & - & TF & Sapien $^{\circledR}$ & 23 \\
\hline $\begin{array}{l}\text { Ribeiro } \\
\text { et al. }{ }^{8}\end{array}$ & 80 & M & $\begin{array}{l}\text { Sem suporte } \\
\text { (stentless) }\end{array}$ & Freestyle ${ }^{\circledR}$ & 23 & Não & 9,7 & 15 & 21 & 28 & 8,2 & TF & Sapien XT ${ }^{\circledR}$ & 23 \\
\hline
\end{tabular}


TABELA 3

Quadro clínico e a conduta

\begin{tabular}{|c|c|c|c|c|c|c|c|c|c|c|c|}
\hline \multirow[b]{2}{*}{ Estudo } & \multirow[b]{2}{*}{$\begin{array}{l}\text { Obstrução } \\
\text { coronária }\end{array}$} & \multicolumn{3}{|c|}{ Quadro clínico } & \multicolumn{2}{|c|}{ Tratamento } & \multirow[b]{2}{*}{$\begin{array}{c}\text { ATC } \\
\text { com } \\
\text { sucesso }\end{array}$} & \multirow[b]{2}{*}{$\begin{array}{l}\text { Tipo } \\
\text { de } \\
\text { stent }\end{array}$} & \multirow[b]{2}{*}{$\begin{array}{c}\text { Necessidade } \\
\text { de suporte } \\
\text { hemodinâmico }\end{array}$} & \multirow[b]{2}{*}{$\begin{array}{c}\text { Tempo de } \\
\text { internação } \\
\text { (dias) }\end{array}$} & \multirow[b]{2}{*}{$\begin{array}{c}\text { Mortalidade } \\
\text { intra- } \\
\text { hospitalar }\end{array}$} \\
\hline & & $\begin{array}{l}\text { Hipotensão } \\
\text { importante }\end{array}$ & $\begin{array}{c}\text { Alterações do } \\
\text { segmento ST }\end{array}$ & $\begin{array}{c}\text { Arritmias } \\
\text { ventriculares } \\
\text { ou RCP }\end{array}$ & ICP & CRM & & & & & \\
\hline Fiorina et al. ${ }^{5}$ & TCE & Sim & Não & Não & $\operatorname{Sim}$ & Não & Sim & $\mathrm{SC}$ & Sim & - & Não \\
\hline Gurvitch et al. ${ }^{6}$ & Ambas & $\operatorname{Sim}$ & Sim & Não & Sim & $\operatorname{Sim}$ & Não & - & Sim & 1 & Sim \\
\hline Gurvitch et al. ${ }^{6}$ & TCE & $\operatorname{Sim}$ & Sim & Sim & Sim & Não & Não & - & Sim & 0 & Sim \\
\hline Chakravarty et al. ${ }^{7}$ & TCE & Sim & Sim & Não & Sim & Não & Sim & SF & Não & - & Não \\
\hline Ribeiro et al. ${ }^{8}$ & TCE & $\operatorname{Sim}$ & Não & Não & Sim & Não & Sim & SF & Não & 3 & Não \\
\hline Ribeiro et al. ${ }^{8}$ & TCE & Sim & Não & $\operatorname{Sim}$ & Sim & Não & Não & - & $\operatorname{Sim}$ & 3 & Sim \\
\hline Ribeiro et al. ${ }^{8}$ & TCE & Sim & Sim & Não & Sim & Não & Sim & SF & Não & 4 & Não \\
\hline
\end{tabular}

RCP = ressuscitação cardiopulmonar; ICP = intervenção coronária percutânea; CRM = cirurgia de revascularização miocárdica; TCE = tronco da artéria coronária esquerda; SC = stent convencional; SF = stent farmacológico.

TABELA 4

Quadro clínico e conduta $(\mathbf{n}=7)$

\begin{tabular}{ll}
\hline Quadro/conduta & \\
\hline Artéria coronária obstruída, $\mathrm{n}(\%)$ & $6(85,7)$ \\
Tronco da artéria coronária esquerda & $1(14,3)$ \\
Ambas as artérias coronárias & \\
Quadro clínico, $\mathrm{n}$ (\%) & $7(100)$ \\
Hipotensão grave persistente & $4(57,1)$ \\
Alterações do segmento ST & $1(14,3)$ \\
Supradesnivelamento do segmento ST & \\
Tratamento, $\mathrm{n}$ (\%) & $7(100)$ \\
Tentativa de intervenção coronária percutânea & $4(57,1)$ \\
Com sucesso & $3(42,9)$ \\
Sem sucesso & $1(14,3)$ \\
$\quad$ Impossibilidade de atravessar a lesão com o fio-guia & $1(14,3)$ \\
$\quad$ Impossibilidade de avançar o stent & $1(14,3)$ \\
$\quad$ Stent implantado mas sem fluxo & \\
Complicações, $n$ (\%) & $4(57,1)$ \\
Necessidade de suporte hemodinâmico & $1(14,3)$ \\
Conversão para cirurgia de revascularização miocárdica & $3(42,9)$ \\
\hline Morte hospitalar
\end{tabular}

plante com sucesso do stent, o que levou a choque cardiogênico persistente e morte; e impossibilidade de avançar o stent. Foi necessário suporte hemodinâmico em quatro pacientes $(57,1 \%)$ e a taxa de mortalidade hospitalar foi $42,9 \%$. Todos os pacientes submetidos a ICP com sucesso sobreviveram e receberam alta hospitalar; nenhum caso apresentou trombose do stent ou nova revascularização.

\section{DISCUSSÃO}

Os principais achados desta revisão sistemática da literatura avaliando a obstrução coronária sintomática após TAVI para o tratamento de disfunção de bioprótese valvular cirúrgica foram que essa complicação ocorreu mais frequentemente em mulheres e em pacientes previamente tratados com próteses sem suporte, ou naquelas com suporte e folhetos montados externamente. Nesses casos, a altura média do óstio do TCE foi $\sim 8 \mathrm{~mm}$ e o diâmetro médio da raiz aórtica foi $\sim 28 \mathrm{~mm}$. O quadro clínico incluiu mais frequentemente hipotensão importante e mantida, além de alterações do segmento ST; uma ICP foi tentada em todos os casos, tendo sucesso em cerca de $60 \%$ deles. Entretanto, suporte mecânico hemodinâmico e conversão para cirurgia de revascularização miocárdica foram ainda necessários em 57\% e em 14\% dos pacientes, respectivamente. Não houve casos de trombose aguda de stent ou de nova revascularização, e a taxa de mortalidade intra-hospitalar foi 42,9\% (não ocorreram óbitos em pacientes submetidos à ICP com sucesso).

A obstrução coronária após TAVI tem sido uma preocupação desde os primeiros estudos experimentais, ${ }^{9,10}$ tendo sido descrita, em 2006, na primeira experiência em seres humanos para o tratamento de estenose de válvula aórtica nativa. ${ }^{11} \mathrm{O}$ primeiro caso de obstrução coronária como uma complicação do procedimento TAVI-ViV foi relatado em 2011. ${ }^{6}$ Desde então, a incidência relatada de obstrução coronária nos diferentes estudos tem sido, geralmente, < 1\%. Entretanto, num grande registro multicêntrico recente sobre obstrução coronária, com mais de 6.500 pacientes, a incidência em válvulas aórticas nativas foi $0,66 \%$, muito mais baixa que os $2,48 \%$ de pacientes com biopróteses cirúrgicas prévias. ${ }^{8}$ Essa incidência é semelhante à de 3,5\% relatada em recente registro realizado em todo o mundo apenas com TAVI-ViV (Registro Global Valve-in-Valve), incluindo 202 pacientes, ${ }^{4}$ e suporta a presença de bioprótese cirúrgica prévia como potencial fator de risco para tal complicação. As taxas de obstrução coronária em recentes estudos avaliando essa complicação, tanto 
em válvulas aórticas nativas quanto em biopróteses cirúrgicas, estão resumidas na Tabela 5 .

\section{Fatores associados à obstrução coronária após o TAVI}

O mecanismo associado à obstrução coronária após TAVI-ViV tem sido o deslocamento do folheto da bioprótese em direção ao óstio coronário em todos os pacientes e nenhum caso de obstrução coronária relacionado à estrutura metálica das válvulas transcateter, nem a seus folhetos, foi relatado até o presente momento. ${ }^{3,8}$ Além disso, de acordo com Registro Global TAVI-ViV, ${ }^{4}$ a obstrução coronária após TAVI-ViV foi mais frequente em biopróteses com suporte e folhetos montados externamente (especialmente com a prótese Mitroflow ${ }^{\circledR}$ ) e também com biopróteses sem suporte. No caso da prótese Mitroflow ${ }^{\circledR}$, os folhetos relativamente longos ( $13 \mathrm{~mm})$ montados externamente sobre os suportes (stent), ao invés de internamente, como ocorre na maioria das outras biopróteses com suporte, podem estar associados a uma taxa mais alta de obstrução coronária. ${ }^{6}$ Além disso, as biopróteses sem suporte (stentless) podem também estar associadas a um maior risco de tal complicação. Essas próteses são geralmente implantadas numa posição supra-anular, resultando num encurtamento da posição dos óstios coronários em relação aos folhetos da válvula, os quais, juntamente da falta das hastes dos stents, podem facilitar a interação da prótese com a parede da aorta e o óstio coronário (Figura 1).

Adicionalmente e em acordo com estudos anteriores sobre TAVI em válvulas aórticas nativas, ${ }^{3,8}$ a presença de óstios coronários, situados numa posição mais baixa, e de seios de Valsalva estreitos também foi identificada como fator de risco em potencial para essa complicação em casos de ViV. Nesse sentido, entre pacientes com dados disponíveis de TC no presente estudo, a altura média do TCE foi de $8,8 \mathrm{~mm}$, semelhante à en- contrada em recente registro multicêntrico $(10,6 \mathrm{~mm})$ e muito mais baixa que os 13,4 mm encontrados em pacientes controles sem obstrução coronária $(P<0,001){ }^{8}$ Essa altura é também significativamente mais baixa $(\sim 5 \mathrm{~mm})$ do que a relatada em estudos anteriores de TC em pacientes com e sem estenose aórtica (sistematicamente $>13 \mathrm{~mm})^{.12-14}$

Embora a altura do óstio coronário seja um fator de risco importante associado à obstrução coronária após o TAVI, demonstrou-se que alguns pacientes tiveram essa complicação a despeito da altura do TCE > $12 \mathrm{~mm}$, indicando outros fatores (como raiz aórtica estreita) que deixavam pouco espaço para acomodar os folhetos aórticos, também como possíveis fatores de risco para a obstrução coronária após o TAVI. ${ }^{3,8}$ De fato, em nosso estudo, o diâmetro médio da raiz aórtica foi de 28,0 mm, comparável ao que foi encontrado em pacientes com obstrução coronária em válvulas aórticas nativas $(28,0 \mathrm{~mm}$ vs. $31,9 \mathrm{~mm}$ nos controles; $\mathrm{P}<0,001){ }^{8}$

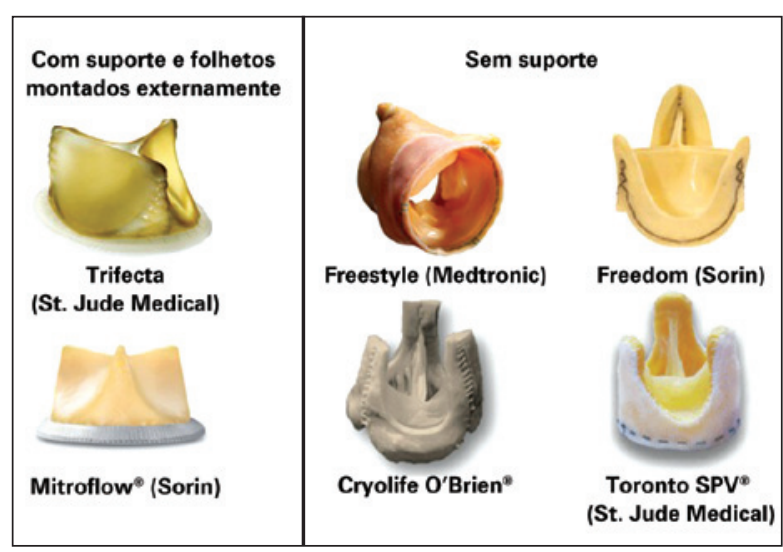

Figura 1. Biopróteses cirúrgicas que têm sido associadas à obstrução coronária após implante transcateter de válvula aórtica.

TABELA 5

Dados de grandes registros de implante transcateter de válvula aórtica que avaliam a obstrução coronária em válvula aórtica nativa e em pacientes com bioprótese cirúrgica prévia

\begin{tabular}{|c|c|c|c|c|c|}
\hline Estudo & $\mathbf{n}$ & Válvula/abordagem & Transfemoral & Transapical & Todos os procedimentos \\
\hline \multirow{4}{*}{$\begin{array}{l}\text { Registro Multicêntrico sobre } \\
\text { Obstrução Coronária }{ }^{8}\end{array}$} & 6.688 & Total & $30 / 4.846(0,62 \%)$ & $13 / 1.531(0,85 \%)$ & $44 / 6.688(0,66 \%)$ \\
\hline & & Sapien $^{\circledast}$ & - & - & $37 / 4.533(0,82 \%)$ \\
\hline & & CoreValve $^{\circledR}$ & - & - & $7 / 2.066(0,34 \%)$ \\
\hline & & Valve-in-valve & - & - & $3 / 121(2,48 \%)$ \\
\hline \multirow[t]{5}{*}{ Registro Global Valve-in-Valvet } & 202 & Total & - & - & $7 / 202(3,5 \%)$ \\
\hline & & Sapien $^{\circledR}$ & - & - & $3 / 78(3,8 \%)$ \\
\hline & & CoreValve $^{\circledR}$ & - & - & $4 / 124(3,2 \%)$ \\
\hline & & Prótese com suporte & - & - & $4 / 155(2,6 \%)$ \\
\hline & & Prótese sem suporte & - & - & $3 / 47(6,4 \%)$ \\
\hline
\end{tabular}


As características clínicas dos pacientes que tiveram obstrução coronária após TAVI-ViV revelaram média de idades (82 anos) e perfil de risco (escore médio STS-PROM de 9,4\%) semelhantes aos relatados na maioria dos estudos anteriores com TAVI. No entanto, até $71 \%$ dos pacientes que tiveram essa complicação foram mulheres, dado um pouco superior à prevalência de $\sim 50 \%$ de mulheres, na maioria dos estudos de TAVI. ${ }^{3}$ Foi demonstrado anteriormente que as mulheres têm, em geral, uma altura do óstio coronário mais baixa e também um menor diâmetro da raiz aórtica, em comparação com os homens, o que poderia explicar, em parte, a maior incidência dessa complicação no sexo feminino. ${ }^{14,15}$ Ademais, tem sido demonstrado que a posição do óstio do TCE é mais baixa em comparação à da artéria coronária direita, o que poderia também explicar porque tal complicação envolve mais frequentemente o TCE. ${ }^{12-14,16,17}$

Em relação às características do procedimento, a maioria dos casos relatados de pacientes que tiveram obstrução coronária após TAVI em válvulas aórticas nativas recebeu uma prótese expansível com balão (Tabela 5). Em nosso estudo, a despeito de termos encontrado mais casos com as válvulas expansíveis por balão, nós não temos toda a população em risco para tirar conclusões definitivas se essa complicação é também mais frequente com válvulas expansíveis por balão, no contexto TAVI-ViV. Além disso, no recente Registro Global TAVI-ViV, o risco de obstrução coronária foi semelhante com ambos os tipos de válvulas (3,2\% para os casos com prótese autoexpansível vs. 3,8\% para os casos expansíveis por balão). Estudos futuros terão que avaliar se há uma interação entre o tipo de válvula e o risco de obstrução coronária, no contexto do TAVI-ViV.

\section{Quadro clínico e conduta na obstrução coronária após TAVI}

Todos os pacientes apresentaram hipotensão importante e mantida após o implante da válvula e cerca de $50 \%$ deles tiveram alterações do segmento ST. Esse quadro clínico poderia ser explicado pelo fato de que o TCE foi mais comumente envolvido. ${ }^{3,8}$ Da mesma maneira, na presença de hipotensão importante e mantida após o implante da válvula, especialmente na presença de alterações do eletrocardiograma, aortografia e/ou ecocardiografia deveriam ser imediatamente realizadas para verificar a presença de obstrução coronária ou nova anormalidade de contração segmentar, respectivamente.

No presente estudo, a ICP foi tentada em todos os pacientes, mas ela não teve sucesso em cerca da metade deles. De maneira importante, o insucesso da ICP foi associado à mortalidade intra-hospitalar em todos os casos e isso levou a uma alta taxa de mortalidade (perto de 50\%). Essa alta taxa de mortalidade é semelhante à relatada em recente registro multicêntrico sobre obstrução coronária $(41 \%)$ e também a relatada no registro global TAVI-ViV $(57,1 \%){ }^{4,8}$ Essas taxas de mortalidade muito altas reforçam o fato de que esse procedimento deve ser realizado em centros altamente experientes, com recursos cirúrgicos, com o intuito de restaurar o fluxo coronário rapidamente, seja por ICP ou mesmo por cirurgia de revascularização miocárdica, em caso de insucesso da ICP. Adicionalmente, como a canulação da artéria coronária pode não ser fácil, em função da presença da estrutura metálica da prótese transcateter, ou mesmo do complexo valvar, especialmente nos pacientes com óstio coronário baixo e seios de Valsalva estreitos, a proteção coronária com um fio-guia (Figura 2) ou mesmo o posicionamento preventivo de um stent coronário poderiam evitar o efeito deletério dessa complicação. ${ }^{7}$

\section{Limitações do estudo}

O presente estudo tem as limitações inerentes a uma revisão sistemática, que coleta apenas as informação descritas nas publicações, de tal sorte que informações relevante eventualmente omitidas nas publicações poderiam acrescentar dados importantes sobre tal complicação para esse subgrupo específico. Além disso, dados de imagens (especialmente de TC) não estavam disponíveis em todos os casos relatados. Finalmente, todos os artigos encontrados na literatura eram relatos de casos ou série de casos pequenas, impedindo a comparação com toda a população sob risco submetida ao TAVI.

\section{CONCLUSÕES}

A obstrução coronária permanece uma complicação rara do TAVI, porém potencialmente fatal e que parece ser mais frequente no contexto de procedimentos valve-in-valve. As características basais dos casos relatados sugerem que essa complicação ocorre mais frequentemente em mulheres, anteriormente submetidas a implante de prótese aórtica sem suporte (stentless) ou de próteses com suporte e folhetos montados externamente. As características inerentes a tais próteses, como longos folhetos, ânulo de sutura raso, posição supra-anular e falta de hastes de suporte, juntamente de característica anatômicas, como óstios em posição baixa e seios de Valsalva de pouca profundidade, podem facilitar a interação da prótese com a parede aórtica e com o óstio coronário. Em relação ao quadro clínico, a ocorrência de hipotensão importante e mantida e de alterações do segmento ST, imediatamente após o implante da válvula, requerem a exclusão dessa complicação. A intervenção coronária percutânea foi possível em $\sim 60 \%$ dos pacientes, com alta taxa de mortalidade, reforçando o fato de que esses procedimentos devem ser realizados apenas em centros altamente especializados e que medidas estratégicas adicionais, como a proteção de um fio-guia, poderiam ser adotadas na presença de parâmetros clínicos ou anatômicos de risco. Futuros estudos prospectivos, incluindo séries consecutivas de pacientes submetidos ao implante transcateter de válvula aórtica valve-in-valve com essa complicação, 

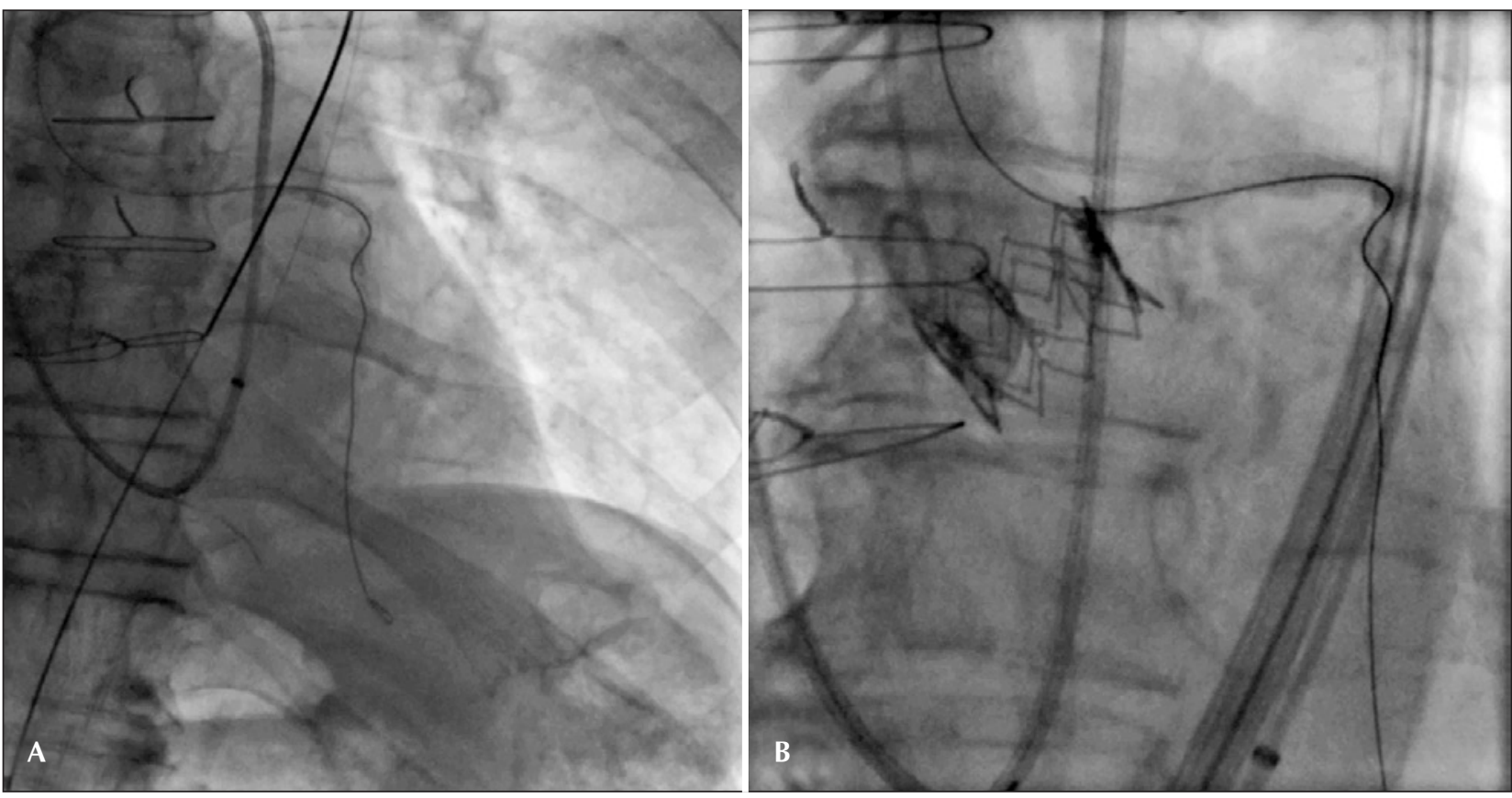

Figura 2. (A) Proteção coronária com fio-guia para facilitar o acesso e a colocação do stent em caso de obstrução coronária após implante transcateter de válvula aórtica para o tratamento de bioprótese com disfunção. (B) Interação entre o fio-guia e a válvula cardíaca transcateter.

são necessários para melhor avaliar fatores preditivos e a conduta clínica mais adequada para essa importante complicação.

\section{SUPORTE FINANCEIRO}

HBR teve apoio de bolsa do Conselho Nacional de Desenvolvimento Científico e Tecnológico (CNPq) para pesquisa de doutorado. LNF teve bolsa de pesquisa da Fundación Mutua Madrileña (Espanha).

\section{CONFLITO DE INTERESSES}

O Dr. Robert DeLarochellière é consultor da St. Jude Medical ${ }^{\circledR}$. O Dr. Éric Dumont é consultor da Edwards Lifesciences. Dr. Josep Rodés-Cabau é consultor da Edwards Lifesciences e da St. Jude Medical ${ }^{\circledR}$. Os demais coautores declaram não ter conflito de interesses.

\section{REFERÊNCIAS}

1. Leon MB, Smith CR, Mack M, Miller DC, Moses JW, Svensson LG, et al. Transcatheter aortic-valve implantation for aortic stenosis in patients who cannot undergo surgery. N Engl J Med. 2010;363(17):1597-607.

2. Smith CR, Leon MB, Mack MJ, Miller DC, Moses JW, Svensson LG, et al. Transcatheter versus surgical aortic-valve replacement in high-risk patients. N Engl J Med. 20119;364(23):2187-98.

3. Ribeiro HB, Nombela-Franco L, Urena M, Mok M, Pasian S, Doyle D, et al. Coronary obstruction following transcatheter aortic valve implantation: a systematic review. JACC Cardiovasc Interv. 2013;6(5):452-61.

4. Dvir D, Webb J, Brecker S, Bleiziffer S, Hildick-Smith D, Colombo A, et al. Transcatheter aortic valve replacement for degenerative bioprosthetic surgical valves: results from the global valve-in-valve registry. Circulation. 2012;126(19):2335-44.

5. Fiorina C, Curello S, Maffeo D, Lorusso R, Chizzola G, Ettori F. Management of acute left main obstruction after transcatheter aortic valve replacement: the "tunnel technique". Cardiovasc Revasc Med. 2012;13(2):142 e5-9.

6. Gurvitch R, Cheung A, Bedogni F, Webb JG. Coronary obstruction following transcatheter aortic valve-in-valve implantation for failed surgical bioprostheses. Catheter Cardiovasc Interv. 2011;77(3):439-44.

7. Chakravarty T, Jilaihawi H, Nakamura M, Kashif M, Kar S, Cheng $\mathrm{W}$, et al. Pre-emptive positioning of a coronary stent in the left anterior descending artery for left main protection: a prerequisite for transcatheter aortic valve-in-valve implantation for failing stentless bioprostheses? Catheter Cardiovasc Interv. 2013;82(4):E630-6.

8. Ribeiro HB, Webb JG, Makkar RR, Cohen MG, Kapadia S, Kodali $S$, et al. Predictive factors, management and clinical outcomes of coronary obstruction following transcatheter aortic valve implantation: insights from a large multicenter registry. J Am Coll Cardiol. 2013;62(17):1552-62.

9. Andersen HR, Knudsen LL, Hasenkam JM. Transluminal implantation of artificial heart valves. Description of a new expandable aortic valve and initial results with implantation by catheter technique in closed chest pigs. Eur Heart J. 1992;13(5):704-8.

10. Flecher EM, Curry JW, Joudinaud TM, Kegel CL, Weber PA, Duran CM. Coronary flow obstruction in percutaneous aortic valve replacement: an in vitro study. Eur J Cardiothorac Surg. 2007;32(2):291-4.

11. Webb JG, Chandavimol M, Thompson CR, Ricci DR, Carere RG, Munt $\mathrm{BI}$, et al. Percutaneous aortic valve implantation retrograde from the femoral artery. Circulation. 2006;113(6):842-50.

12. Tops LF, Wood DA, Delgado V, Schuijf JD, Mayo JR, Pasupati $S$, et al. Noninvasive evaluation of the aortic root with multis- 
lice computed tomography implications for transcatheter aortic valve replacement. JACC Cardiovasc Imaging. 2008;1(3):321-30.

13. Akhtar M, Tuzcu EM, Kapadia SR, Svensson LG, Greenberg RK, Roselli EE, et al. Aortic root morphology in patients undergoing percutaneous aortic valve replacement: evidence of aortic root remodeling. J Thorac Cardiovasc Surg. 2009; 137(4):950-6.

14. Buellesfeld L, Stortecky S, Kalesan B, Gloekler S, Khattab AA, Nietlispach $F$, et al. Aortic root dimensions among patients with severe aortic stenosis undergoing transcatheter aortic valve replacement. JACC Cardiovasc Interv. 2013;6(1):72-83.
15. Vasan RS, Larson MG, Levy D. Determinants of echocardiographic aortic root size. The Framingham Heart Study. Circulation. 1995;91(3):734-40.

16. Apfaltrer P, Schymik G, Reimer P, Schroefel H, Sueselbeck $\mathrm{T}$, Henzler $\mathrm{T}$, et al. Aortoiliac CT angiography for planning transcutaneous aortic valve implantation: aortic root anatomy and frequency of clinically significant incidental findings. AJR Am J Roentgenol. 2012;198(4):939-45.

17. Cavalcanti JS, Melo NC, Vasconcelos RS. Morphometric and topographic study of coronary ostia. Arq Bras Cardiol. 2003; 81(4):359-62, 355-8. 